Forum 2017 · 32:454

https://doi.org/10.1007/s12312-017-0348-1

○) Springer Medizin Verlag GmbH 2017

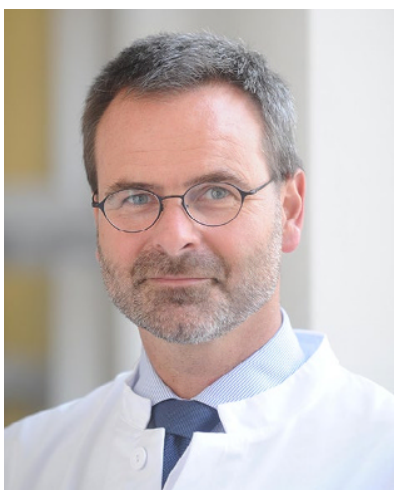

Der Wert klinischer Studien für den medizinischen Fortschritt ist unbestritten. Sie liefern die Evidenzgrundlage für medizinische Leitlinien und damit die Basis für Qualitätsmanagement im Gesundheitswesen. Hochwertig sollen sie sein, also möglichst randomisiert kontrolliert und prospektiv geplant. Diese Anforderungen sind nicht zum Nulltarif umzusetzen. Kein Wunder also, dass der überwiegende Teil dieser Studien industriefinanziert ist. Dabei schneidet Deutschland im internationalen Vergleich offenbar nicht so schlecht ab. Laut eines vfa-Berichts starteten 2016 in den USA 2306 klinische Studien auf Initiative von Unternehmen, Deutschland folgt mit 532 Industrie-Studien auf Platz 2 [1]. Allein 25\% dieser Studien entfielen auf die Erforschung neuer Krebstherapien und -diagnostika.

Ohne Frage gäbe es die meisten medizinischen Innovationen nicht ohne die Industrie. Dennoch können wirtschaftliche Interessen dazu führen, dass negative Ergebnisse klinischer Studien nicht oder mit deutlicher Verzögerung publiziert werden. Experten weisen schon lange auf den entstehenden Publikationsbias hin. In der Chirurgie oder der Allgemeinmedizin ist das Forschungsinteresse gewerblicher Unternehmen eher gering. Das Gleiche gilt für Head-to-Head-Vergleiche verschiedener Behandlungsoptionen. Und bei speziellen Krankheitssituationen, etwa der

\title{
Mehr Transparenz bei der Vergabe öffentlicher Fördergelder
}

Versorgung von Patienten am Lebensende, herrscht ein erschreckender Mangel an Studiendaten. Hier ist unabhängige Forschungsförderung gefragt.

In der "Strategie Europa 2020“ haben sich alle beteiligten EU-Länder darauf verständigt, drei Prozent des Bruttoinlandsprodukts für Forschung und Entwicklung (FuE) auszugeben. Deutschland erreicht zwar dieses Ziel. Aber von insgesamt 84 Milliarden Euro für FuE im Jahr 2014 stammen ein Drittel vom Staat und zwei Drittel aus der Wirtschaft [2]. Insgesamt 2.5 Milliarden Euro sind 2017 von der öffentlichen Hand für die Gesundheitsforschung eingeplant, das entspricht ungefähr $10 \%$ der öffentlichen FuE-Ausgaben. Doch wie viel davon kommt dort an, wo es benötigt wird?

Gemeinnützigen Förderern wie der Deutschen Krebshilfe ist es zu verdanken, dass onkologische Forschungsprojekte und Therapiestudien angestoßen werden - finanziert aus Spendengeldern. Das BMBF, praktisch der größte öffentliche FuE-Förderer im deutschen Gesundheitsbereich, weist dagegen im Haushalt 2016 für die gesamte Förderung klinischer Studien vergleichsweise bescheidene Mittel aus, ca. 50 Millionen Euro [3]. Zum Vergleich: In England stehen jährlich knapp 300 Millionen Pfund aus öffentlichen Geldern für Studien zur Verfügung.

Immerhin ist die Notwendigkeit von Versorgungsforschung mittlerweile ins öffentliche Bewusstsein gerückt - der Innovationsfonds fördert hier für drei Jahre mit 75 Millionen Euro jährlich, dazu kommen 15 Millionen vom BMBF. Das freut uns einige der geförderten onkologischen Projekte werden in diesem Heft vorgestellt.

Dennoch erstaunt es, wie wenig koordiniert die Forschungsförderung in Deutschland erscheint. Es fehlt an Transparenz darüber, welche übergeordneten Gesundheitsziele erreicht werden sollen, welche Forschungsfragen zur Zielerreichung geklärt, welche Evidenzlücken dringlich gestopft werden müssen und welchen Benefit die Förderung verspricht. Das gilt im Übrigen auch für die Versorgungsforschung durch den Innovationsfonds - auch hier vermisst man die Zielrichtung sowie eine Entscheidung durch neutrale Gutachter.

Wenn relevante klinische Forschungsfragen die Hürden des durch unterschiedliche politische Interessen geprägten G-BA passieren müssen, dann wird häufig nur der kleinste Nenner realisierbar sein. Gerade deshalb ist es wichtig, dass klinische Forschung durch klinisch tätige Experten entwickelt und durch die besten unabhängigen Gutachter beurteilt wird. Wenn Leistungsträger Evidenz in wichtigen Therapieentscheidungen fordern, dann müssen sie die Generierung dieser Evidenz auch finanzieren und können dies nicht privaten Sponsoren oder der Industrie überlassen.

Ihr

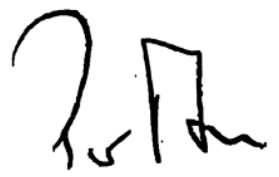

Peter Albers

Präsident der Deutschen Krebsgesellschaft

\section{Literatur}

1. Pressemitteilung vfa vom 15.06.2017. https:// www.vfa.de/de/presse/pressemitteilungen/ pm-010-2017-deutschland-erneut-weltweit-nr.2-bei-klinischen-studien-forschender-pharmaunternehmen.html. Zugriff: 03. November 2017

2. Gemeinsame Wissenschaftskonferenz, Sachstandsbericht zum Erreichen des 3\%-Ziels an die Regierungschefinnen und Regierungschefs von Bund und Ländern 2017. https://www.gwk-bonn. de/fileadmin/Papers/GWK-Heft-51-StrategieEuropa-2020.pdf. Zugriff: 03. November 2017

3. Bundesministerium für Bildung und Forschung Bildung und Forschung in Zahlen 2017. https:// www.bmbf.de/pub/Bildung_und_Forschung_in_ Zahlen_2017.pdf. Zugriff: 03. November 2017 